\title{
Doppler Velocity Estimation of Overlapping Linear-Period-Modulated Ultrasonic Waves Based on an Expectation-Maximization Algorithm
}

\author{
Natee Thong-un and Minoru K. Kurosawa \\ Department of Information Processing, Interdisciplinary Graduate School of Science and Engineering, Tokyo Institute of \\ Technology, 4259 Nagatsutamati, Midori-ku, Yokohama, Kanagawa 226-8502, Japan
}

Correspondence should be addressed to Natee Thong-un; thnatee@yahoo.co.th

Received 30 April 2014; Revised 8 October 2014; Accepted 15 October 2014; Published 12 November 2014

Academic Editor: Benjamin Soenarko

Copyright (c) 2014 N. Thong-un and M. K. Kurosawa. This is an open access article distributed under the Creative Commons Attribution License, which permits unrestricted use, distribution, and reproduction in any medium, provided the original work is properly cited.

\begin{abstract}
The occurrence of an overlapping signal is a significant problem in performing multiple objects localization. Doppler velocity is sensitive to the echo shape and is also able to be connected to the physical properties of moving objects, especially for a pulse compression ultrasonic signal. The expectation-maximization (EM) algorithm has the ability to achieve signal separation. Thus, applying the EM algorithm to the overlapping pulse compression signals is of interest. This paper describes a proposed method, based on the EM algorithm, of Doppler velocity estimation for overlapping linear-period-modulated (LPM) ultrasonic signals. Simulations are used to validate the proposed method.
\end{abstract}

\section{Introduction}

Recently, acoustic systems have been used in many industrial and educational applications because such systems have the advantages of a variety of acoustic transducers, low cost, small size, and simple hardware. For example, ultrasonic distance measurement is a modern and relatively flexible approach for environment recognition. The ultrasonic distance measurement determination uses the time-of-flight (TOF) approach, which is the time for sound waves to travel between the sound source and the objects. In general, TOF can simply be computed by performing a cross-correlation between the transmitted and received signals [1]. As a result, TOF can be measured by the maximum peak during a storage time.

A linear-frequency-modulated (LFM) signal, which has a linear variation in an instantaneous frequency with time, is an example of a pulse compression signal used for ultrasonic distance measurement [1]. However, for the case of the distance measurement of the moving object, the LFM signal is unsuitable because of the shifted frequency of the Doppler effect. The problem is that the cross-correlation cannot completely be achieved between the transmitted and received signals. Therefore, a linear-period-modulated (LPM) signal, the period of which is linearly swept with time, has been demonstrated to solve the Doppler-shift problem of the LFM signal $[2,3]$. A LPM signal can be used in the crosscorrelation by providing the TOF with the Doppler-shift compensation via Doppler velocity estimation [4].

However, the Doppler-shift problem is more challenging for the multiple-echoes case. The overlapping signals due to many objects will exhibit many Doppler velocities. If each Doppler velocity can be completely separated, then the Doppler velocity can be estimated for each individual echo. In the case of a pulse compression signal, the LFM echoes can be discriminated by the use of the Fractional Fourier Transform $[5,6]$. The Fractional Fourier Transform method can identify the time delay and the length of each echo. The length of the echo can be used to determine the Doppler-shifted frequency of the reflectors, which is directly related to the Doppler velocity. However, the Fractional Fourier Transform cannot be adapted for use with a nonlinear frequency modulated signal, such as the LPM signal. Therefore, this paper describes the proposed method of estimating the Doppler velocities embedded in the overlapping LPM signals. This proposed 
idea is based on the expectation-maximization (EM) algorithm. The EM algorithm [7], although iterative in nature, is guaranteed under certain mild conditions to converge and, at convergence, to produce at least a local maximum. The EM algorithm has the desirable property of increasing the likelihood of finding a local maximum at each step. Moreover, the EM algorithm has been used to estimate the unknown parameters in the overlapping Gaussian signals [8]. Note that, for the overlapping LPM ultrasonic signals, the EM algorithm is interesting in that this method can estimate the unknown parameters of each signal. Simulation results indicate that the EM algorithm can provide the Doppler velocity estimation of the overlapping LPM ultrasonic signals.

\section{Doppler Velocity Estimation for a Single LPM Echo}

The discrete signal of the LPM ultrasonic signal can be modeled as [3]

$$
f(n)=A \sin \left[2 \pi \frac{l_{0}}{p_{b}} \ln \left\{\frac{p_{b}}{p_{1} l_{0}} n+1\right\}\right]
$$

for $n=0,1,2, \ldots, N-1$ where $A$ is the rectangular envelope pulse of the amplitude in a wide band frequency, $p_{b}$ is the sweep period band of the reference LPM ultrasonic signal, $p_{1}$ is the starting period time, $l_{0}$ is the length of the LPM ultrasonic signal, and $N$ is the total number of samples. When we consider the LPM ultrasonic echo produced by reflection from the moving object, it can be expressed in a form of the noisy signal as

$$
\begin{gathered}
x(n)=s(n)+\eta(n), \\
s(n)=B \sin \left[2 \pi \frac{l_{0}}{p_{b}} \ln \left\{\alpha\left(v_{d}\right) \frac{p_{b}}{p_{1} l_{0}}\left(n-n_{\mathrm{TOF}}\right)+1\right\}\right],
\end{gathered}
$$

where $n_{\mathrm{TOF}}$ is a sample when TOF happens; $\alpha\left(v_{d}\right)=(v+$ $\left.v_{d}\right) / v$ is the Doppler-shift function of the modulated LPM ultrasonic signal expressed in [4], where $v$ is the ultrasonic velocity in air; and $v_{d}$ is the object velocity. $\alpha\left(v_{d}\right)$ is directly related to the length of the received LPM ultrasonic echo. The amplitude of the received LPM ultrasonic echo accounting for attenuation along the distance travelled is $B=A 10^{-2.11} \mathrm{vnTOF}$ [1]. To verify the ability to estimate the Doppler velocity for a noisy situation, noise can be added to the model. The noise $\eta(n)$ is represented as the additive white Gaussian noise (WGN). In this case, to estimate the Doppler velocity $\left(v_{d}\right)$, because no overlapping signals are present, it is not necessary to use the E-step of the EM algorithm. The estimation can be only performed in the maximization, $M$-step. In the statistical model, we assume that the $x(n)$ of each sample is independent and has an identical distribution. Then, their probability density function (pdf) is the normal distribution illustrated as

$$
p\left(x ; v_{d}\right)=\prod_{n=0}^{N-1} \frac{1}{\sqrt{2 \pi \sigma^{2}}} \exp \left[-\frac{1}{2 \sigma^{2}}\left(x(n)-s\left(n ; v_{d}\right)\right)^{2}\right]
$$

where $\sigma^{2}$ is the variance of the Gaussian distribution and $x(n)$ is the sampling data $[x(0), x(1), \ldots, x(N-1)]^{T}$. It can be expressed in a form of the noisy signal as

$$
\begin{aligned}
& \eta(n) ; \quad 0<n \leq n_{\mathrm{TOF}}-1 \\
& x(n)=s\left(n-n_{\mathrm{TOF}}\right)+\eta(n) ; \quad n_{\mathrm{TOF}} \leq n \leq n_{\mathrm{TOF}}+L_{d}-1 \\
& \eta(n) ; \quad n_{\mathrm{TOF}}+L_{d} \leq n \leq N-1 .
\end{aligned}
$$

The pdf of (3) can be expanded to

$$
\begin{aligned}
p\left(x ; v_{d}\right)= & \prod_{n=0}^{n_{\mathrm{TOF}}-1} \frac{1}{\sqrt{2 \pi \sigma^{2}}} \exp \left[-\frac{1}{2 \sigma^{2}} x^{2}(n)\right] \\
& \cdot \prod_{n=n_{\mathrm{TOF}}}^{n_{\mathrm{TOF}}+L_{d}-1} \frac{1}{\sqrt{2 \pi \sigma^{2}}} \\
& \times \exp \left[-\frac{1}{2 \sigma^{2}}\left(x(n)-s\left(n-n_{\mathrm{TOF}}\right)\right)^{2}\right] \\
& \cdot \prod_{n=n_{\mathrm{TOF}}+L_{d}}^{N-1} \frac{1}{\sqrt{2 \pi \sigma^{2}}} \exp \left[-\frac{1}{2 \sigma^{2}} x^{2}(n)\right] .
\end{aligned}
$$

The maximum likelihood estimation (MLE) function is simplified to

$$
\begin{array}{r}
p\left(x ; v_{d}\right)=\frac{1}{\left(2 \pi \sigma^{2}\right)^{N / 2}} \exp \left[-\frac{1}{2 \sigma^{2}} \sum_{n=0}^{N-1} x^{2}(n)\right] \\
\cdot \prod_{n=n_{\mathrm{TOF}}}^{n_{\mathrm{TOF}}+L_{d}-1} \exp \left[-\frac{1}{2 \sigma^{2}}\left(-2 x(n) s\left(n-n_{\mathrm{TOF}}\right)\right.\right. \\
\left.\left.+s^{2}\left(n-n_{\mathrm{TOF}}\right)\right)\right] .
\end{array}
$$

The MLE is found by only maximizing $\exp [-(1 /$ $\left.\left.2 \sigma^{2}\right) \sum_{n=n_{\mathrm{TOF}}}^{n_{\mathrm{TOF}}+L_{d}-1}\left(-2 x(n) s\left(n-n_{\mathrm{TOF}}\right)+s^{2}\left(n-n_{\mathrm{TOF}}\right)\right)\right]$ or, equivalently, by minimizing

$$
\sum_{n=n_{\mathrm{TOF}}}^{n_{\mathrm{TOF}}+L_{d}-1}\left[-2 x(n) s\left(n-n_{\mathrm{TOF}}\right)+s^{2}\left(n-n_{\mathrm{TOF}}\right)\right] .
$$

Then $L_{d}=\left(v / v+v_{d}\right) L_{0}$ [4], where $L_{d}$ and $L_{0}$ are the lengths of Doppler shift and reference signals, respectively. When carefully scrutinizing in (7), we note that $\sum_{n=n_{\mathrm{TOF}}}^{n_{\mathrm{TOF}}+L_{d}-1} s^{2}\left(n-n_{\mathrm{TOF}}\right)$ does not deal with the estimation between the measured data $x(n)$ and the model of the LPM ultrasonic signal $s\left(n ; v_{d}\right)$; therefore, we can ignore it. Therefore, the MLE of $n_{\mathrm{TOF}}$ is expressed by maximizing

$$
\sum_{n=n_{\mathrm{TOF}}}^{n_{\mathrm{TOF}}+L_{d}-1}\left[x(n) s\left(n-n_{\mathrm{TOF}}\right)\right]
$$




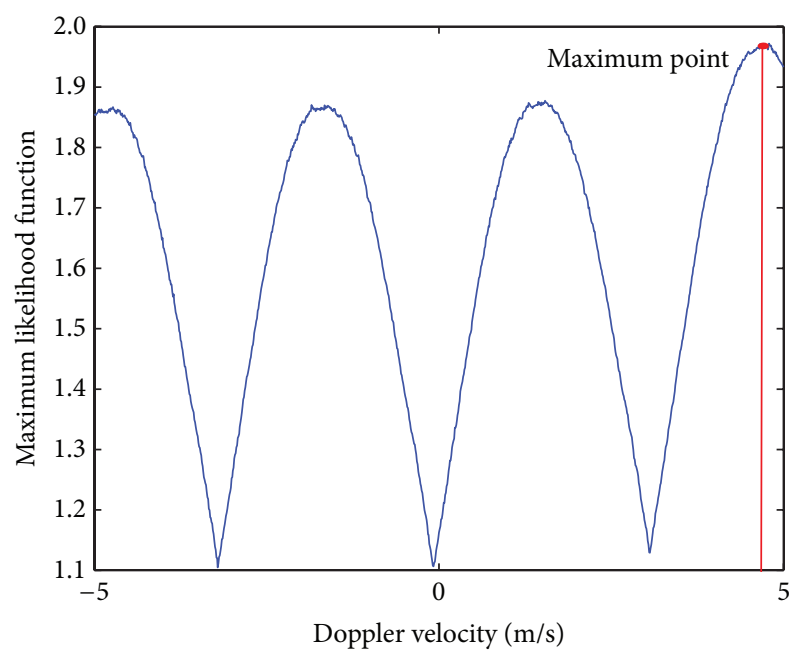

(a)

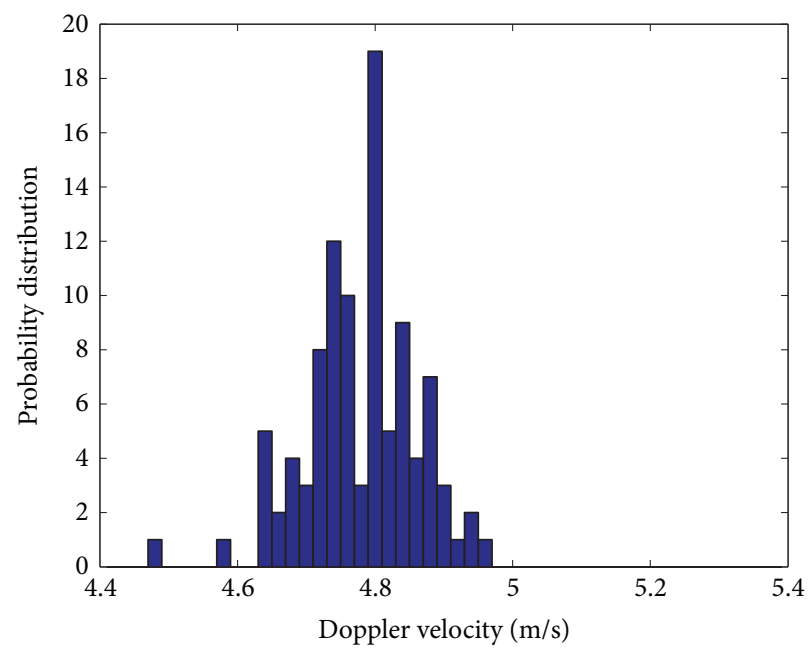

(c)

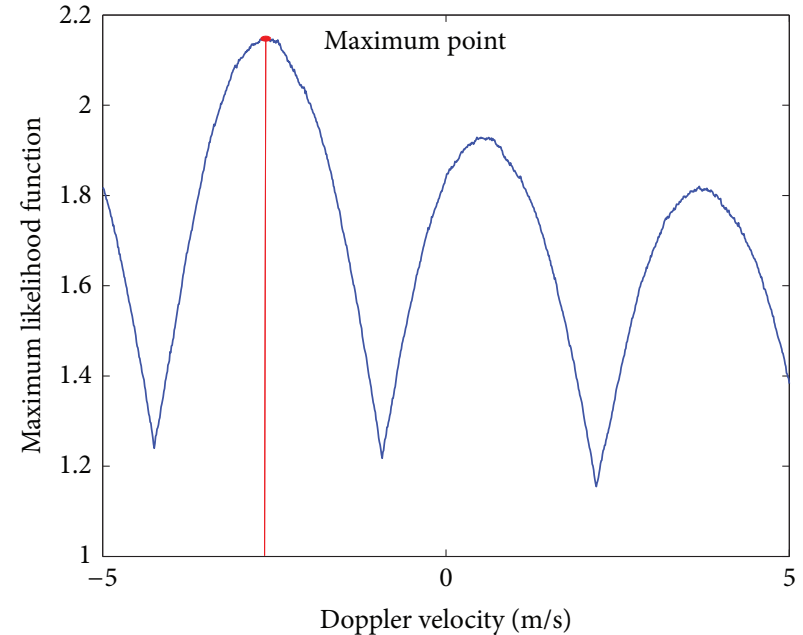

(b)

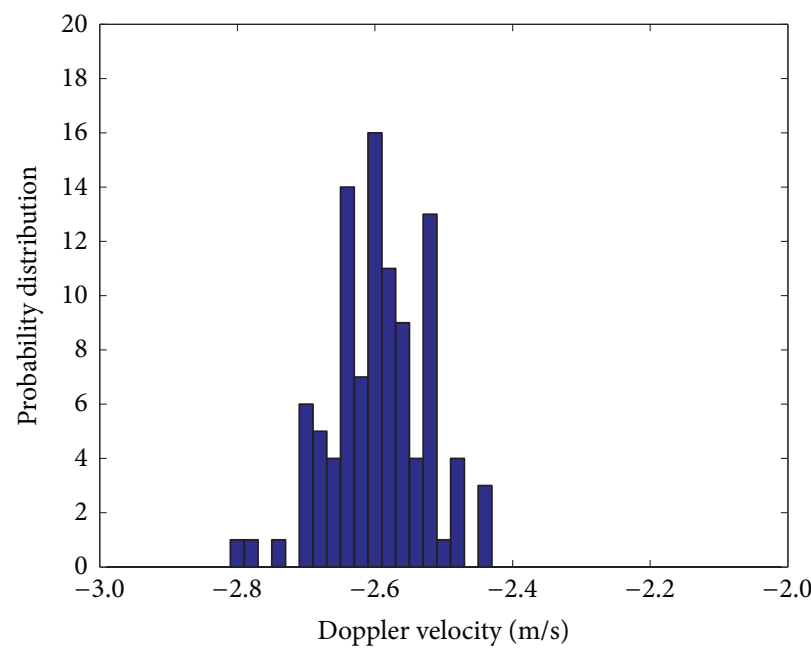

(d)

FIGURE 1: Estimation searching and probability distribution of the Doppler velocity.

Simply speaking, (8) is the correlation between the measured data $x(n)$ and the model signal $s(n)$. Therefore, (8) provides the maximum likelihood function with the exact Doppler velocity under the peak value of the cross-correlation. It can be defined as

$$
\widehat{v}_{d}=\underset{v d \in \Theta}{\arg \max } \sum_{n=n_{\mathrm{TOF}}}^{n_{\mathrm{TOF}}+L_{d}-1}\left[x(n) s\left(n-n_{\mathrm{TOF}}\right)\right],
$$

where $\Theta$ is the region of interest and $v_{d}$ is the Doppler velocity estimator.

This idea was evaluated by performing a MATLAB computer simulation. The period of the transmitted LPM ultrasonic signal was swept from $20 \mu$ s to $50 \mu \mathrm{s}$. The sampling frequency rate was $12.5 \mathrm{MHz}$. A pulse length was equal to $3.247 \mathrm{~ms}$. In the environment setup, the propagation velocity of an ultrasonic wave in air was $343.6426 \mathrm{~m} / \mathrm{s}$ at temperature $20^{\circ} \mathrm{C}$, and the attenuation was set at $2.11 \mathrm{~dB} / \mathrm{m}$ along the travelling distance. In the simulation, the Doppler velocity of the interesting region was varied from $-5 \mathrm{~m} / \mathrm{s}$ to $5 \mathrm{~m} / \mathrm{s}$, and the instantaneous position of the moving object far away from the speaker was $1 \mathrm{~m}$. The grid-search method was used for determining the Doppler velocity via the maximum likelihood function. The search had a step resolution equivalent to $0.01 \mathrm{~m} / \mathrm{s}$. Figure 1 shows the maximum Doppler velocity estimation, which was computed from (9), when the actual values of which were assumed that an object was moving in and out to a sound source with 4.8 and $-2.6 \mathrm{~m} / \mathrm{s}$, respectively. For each Doppler velocity, 100 trials were performed to determine the probability distribution. The SNR of the setup with randomness was $0 \mathrm{~dB}$.

\section{Doppler Velocity Estimation for Overlapping LPM Echoes}

In the previous section, the Doppler velocity estimation of a LMP ultrasonic echo can be performed. However, 


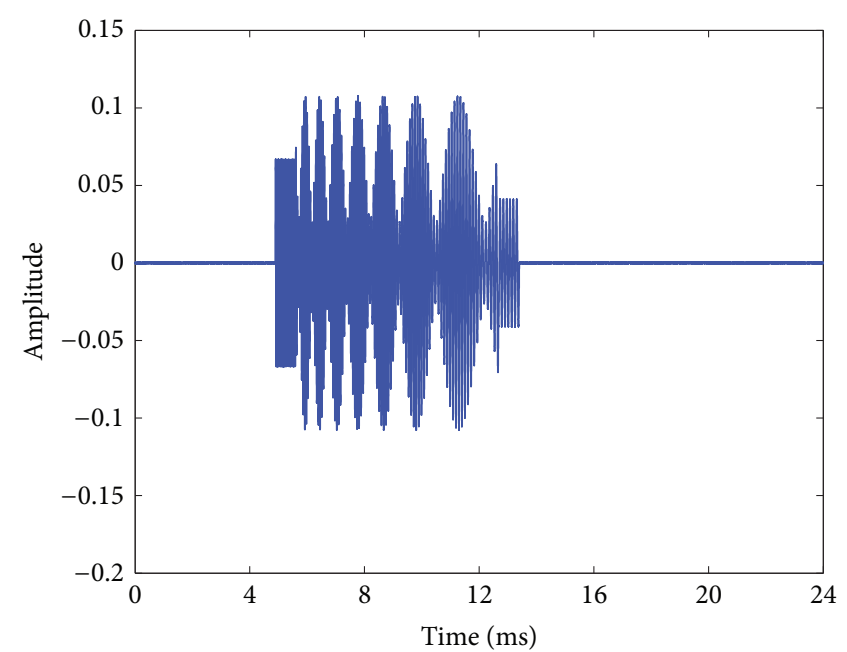

(a)

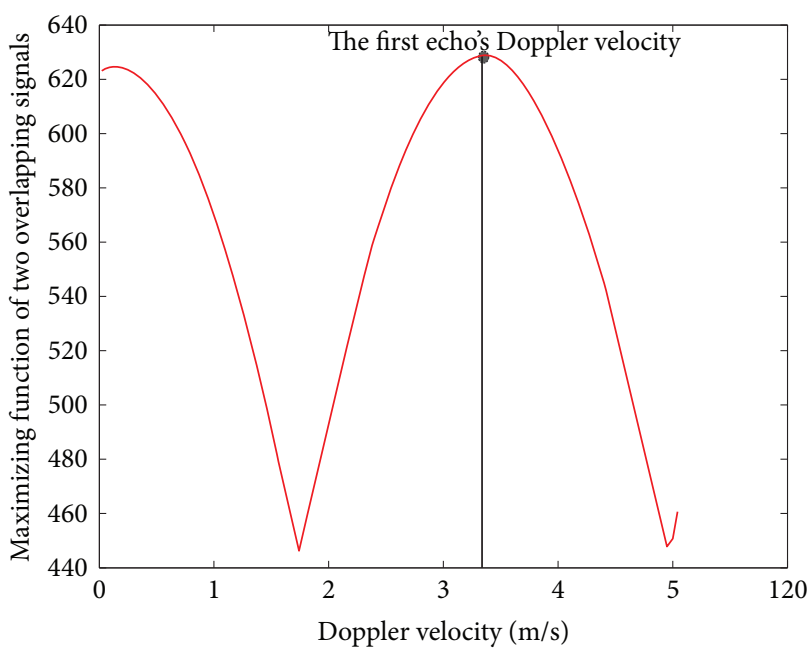

(b)

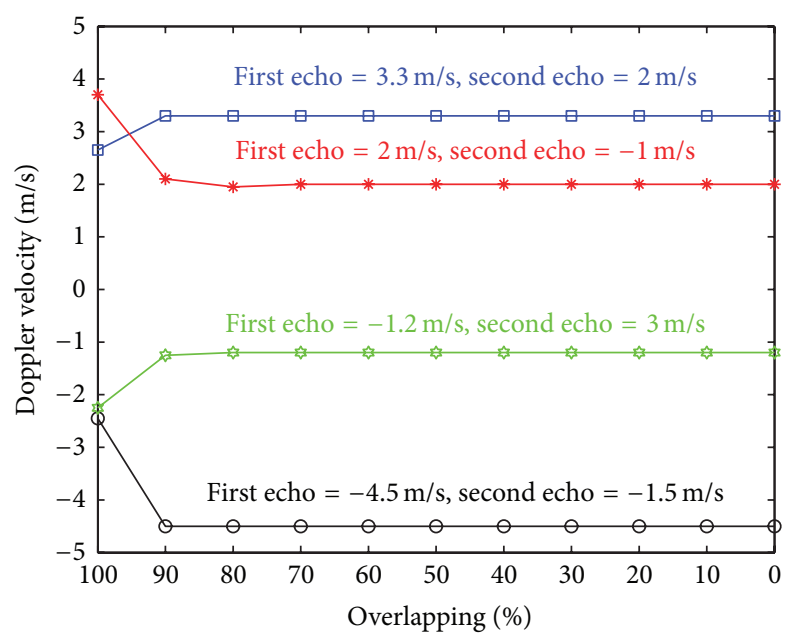

(c)

Figure 2: (a) Two overlapping LPM ultrasonic echoes at distances of $0.7 \mathrm{~m}$ and $0.75 \mathrm{~m}$ from the sound source. (b) Maximizing function of two overlapping echoes: the first and second echoes with $3.3 \mathrm{~m} / \mathrm{s}$ and $2 \mathrm{~m} / \mathrm{s}$, respectively. (c) Percentage of overlap.

the estimation will be more complicated if M-number of overlapping echoes are present. To proceed further, considering the overlapping signals, the LPM discrete signal is

$$
y(n)=\sum_{m=1}^{M} s_{m}(n)+\eta(n)
$$

where $s_{m}(n)$ is the overlapping LPM ultrasonic echo model and $y(n)$ is the measured echo. In general, the amplitudes under the overlapping echoes are directly related to their distance; therefore, the other echoes except for the first echo can be treated as noise. When the observations $y(n)$ are independent and exhibit identical distributions, their probability density function (pdf) is the normal distribution according to

$$
p\left(y ; v_{d 1}\right)=\prod_{n=0}^{N-1} \frac{1}{\sqrt{2 \pi \sigma^{2}}} \exp \left[-\frac{1}{2 \sigma^{2}}\left(y(n)-s_{1}(n)\right)^{2}\right]
$$

or equivalently maximizing

$$
\widehat{v}_{d 1}=\underset{v d \in \Theta}{\arg \max } \sum_{n=n_{\mathrm{TOF} 1}}^{n_{\mathrm{TOF} 1}+L_{d 1}-1}\left[y(n) s_{1}\left(n-n_{\mathrm{TOF} 1}\right)\right] .
$$

Equations (11) and (12) represent the correlation of the measured data with the first echo. Assume that two overlapping signals are at distances of $0.7 \mathrm{~m}$ and $0.75 \mathrm{~m}$ from the sound source, which is pictured in Figure 2(a). The first echo has a Doppler velocity of $3.3 \mathrm{~m} / \mathrm{s}$, and the second echo has a Doppler velocity of $2 \mathrm{~m} / \mathrm{s}$. The maximum likelihood function of the Doppler velocity of the first echo is shown in Figure 2(b). In addition, Figure 2(c) shows the ability of the model to estimate the Doppler velocity of the first echo, in which the second echo is embedded, when the amount of overlap is changed. With the estimate of the Doppler velocity of the first echo, the Doppler velocity of the residual echo now must be estimated. To accomplish this estimation, the EM algorithm allows us to separate the overlapping echoes 


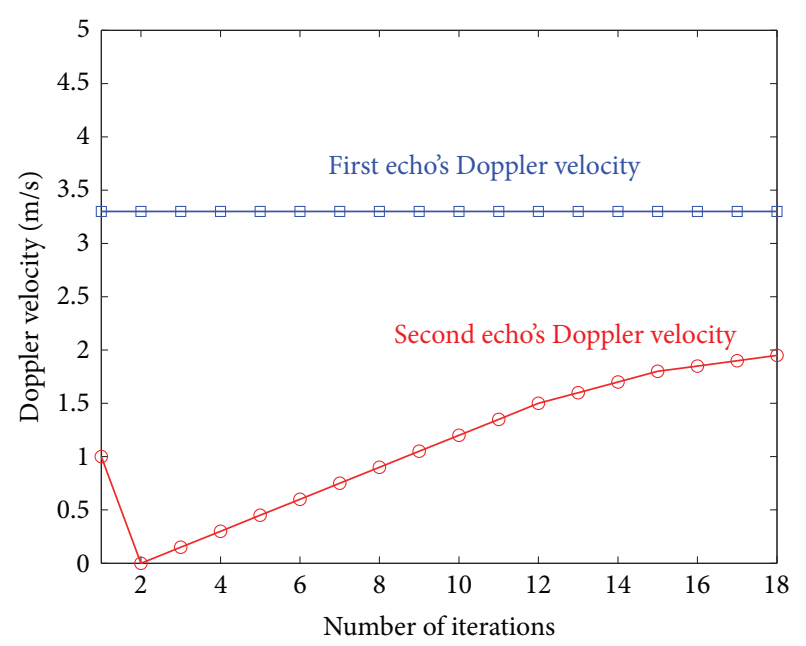

(a)

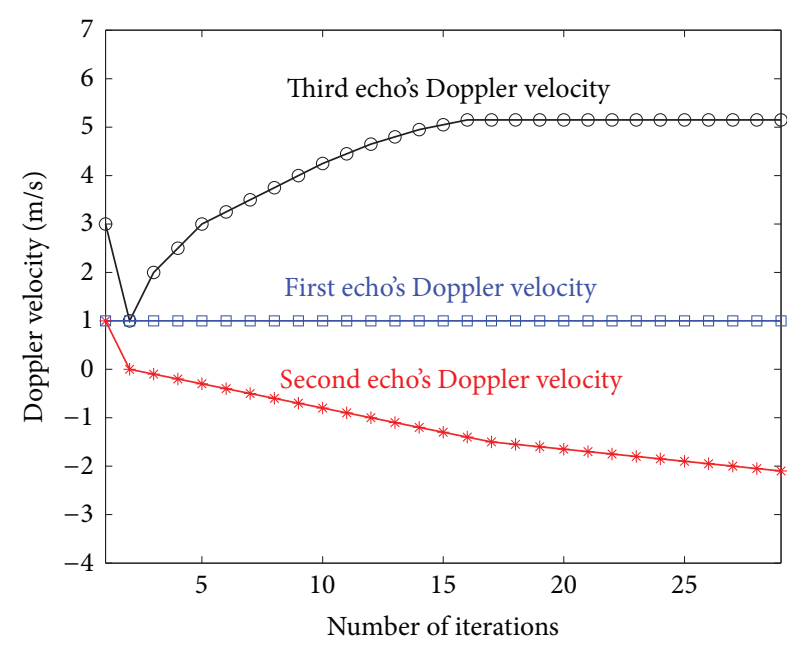

(b)

FIGURE 3: Iterations for overlapping LPM ultrasonic echoes with a $0.05 \mathrm{~m} / \mathrm{s}$ step of the grid-search method. (a) Two overlapping LPM ultrasonic echoes with $v_{d 1}$ and $v_{d 2}$ of $3.3 \mathrm{~m} / \mathrm{s}$ and $2 \mathrm{~m} / \mathrm{s}$, respectively. (b) Three overlapping LPM ultrasonic echoes with $v_{d 1}, v_{d 2}$, and $v_{d 3}$ of $1 \mathrm{~m} / \mathrm{s},-2 \mathrm{~m} / \mathrm{s}$, and $5 \mathrm{~m} / \mathrm{s}$, respectively.

into the individual echoes by means of the expectation and maximization steps. First, we assume that $y_{m}$ is characterized as the unknown data for at $m$ th LPM ultrasonic echo under the WGN process $\eta_{m}(n)$ as

$$
y_{m}(n)=s_{m}(n)+\eta_{m}(n) .
$$

The unknown data of each individual echo can be formed as a linear transformation of the measured data $y=\sum_{m=1}^{M} y_{m}$. The $E$-step of the EM algorithm is able to compute $y_{m}$ in terms of the measured and estimated data as

$$
\widehat{y}_{m}^{k}(n)=s_{m}^{k}(n)+\beta_{m}\left(y(n)-\sum_{i=1}^{M} s_{i}^{k}\right),
$$

where $\sum_{m=1}^{M} \beta_{m}=1$; for the example of simulation, if there are the two overlapping echoes, $\beta_{1}$ and $\beta_{2}$ are $0.5 . k$ represents the number of iterations, which simply keeps a balance of the observation and the estimation of every echo. The expected data $\widehat{y}_{m}$ is adjusted according to the difference between the observation and the estimation. Then, the $M$-step of the EM algorithm, which involves the maximization of the pdf, is expressed as

$$
\widehat{v}_{d m}^{k+1}=\underset{v d m}{\arg \max } \sum_{n=0}^{N-1} \widehat{y}_{m} \cdot s_{m}\left(n-n_{\mathrm{TOFm}}\right) .
$$

The definition can be concluded in the form of an algorithm for the Doppler velocity estimation of the $M$-number of the overlapping LPM ultrasonic echoes in Algorithm 1.

Algorithm 1. (1) Use the Maximum likelihood estimation ( $M$ step) to estimate the Doppler velocity of the first echo and set the starting iteration $k=0$.

(2) Make the initial guesses of the residual echoes $v_{d 2}^{0}$, $v_{d 3}^{0}, \ldots, v_{d M}^{0}$.
(3) For $m=2,3, \ldots, M$, compute the expected echoes ( $E$ step):

$$
\widehat{y}_{m}^{k}(n)=s_{m}^{k}(n)+\beta_{m}\left(y(n)-\sum_{i=1}^{M} s_{i}^{k}\right) .
$$

(4) For $m=2,3, \ldots, M$, compute the maximization ( $M$ step):

$$
\widehat{v}_{d m}^{k+1}=\underset{v d m}{\arg \max } \sum_{n=0}^{N-1} \hat{y}_{m} \cdot s_{m}\left(n-n_{\mathrm{TOF} m}\right) .
$$

(5) Adjust $v_{d 2}^{k}, v_{d 3}^{k}, \ldots, v_{d M}^{k}$ for the update:

$$
\begin{aligned}
s_{m=2,3, \ldots, M}^{k+1}(n)=B \sin \left[2 \pi \frac{l_{0}}{p_{b}} \ln \{\right. & \alpha\left(v_{d m=2,3, \ldots, M}^{k}\right) \\
& \left.\left.\times \frac{p_{b}}{p_{1} l_{0}}\left(n-n_{\mathrm{TOF} m}\right)+1\right\}\right] .
\end{aligned}
$$

(6) Check the convergence criterion: if $\left\|\widehat{v}_{d m}^{k+1}-\widehat{v}_{d m}^{k}\right\| \leq$ tolerance, then stop.

(7) Set $k \rightarrow k+1$ and back to step 3 .

Note that, in this algorithm, the grid search is a main approach for each echo. Iterations for convergence of two and three overlapping LPM ultrasonic echoes are shown Figure 3.

\section{Simulation Results and Discussion}

The algorithm was tested by performing simulations of the LPM ultrasonic echoes. In the case of one Doppler velocity estimation, the Doppler velocity and distance to the moving object were determined under 100 times according to support statistics. The probability distribution of 


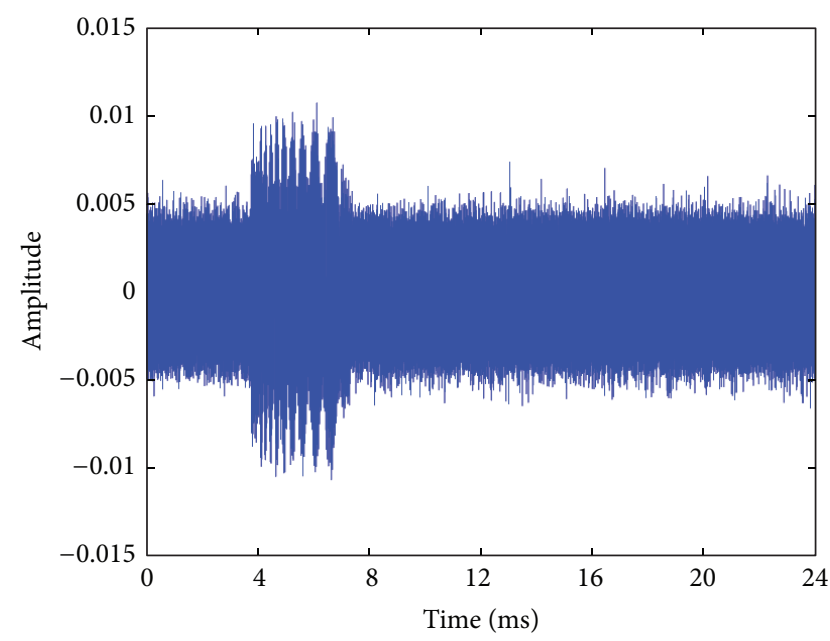

(a)

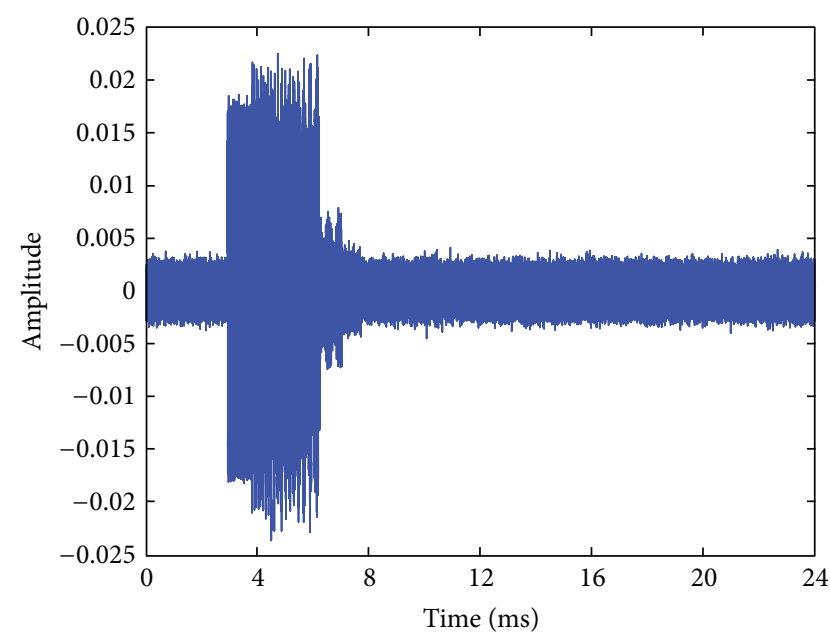

(c)

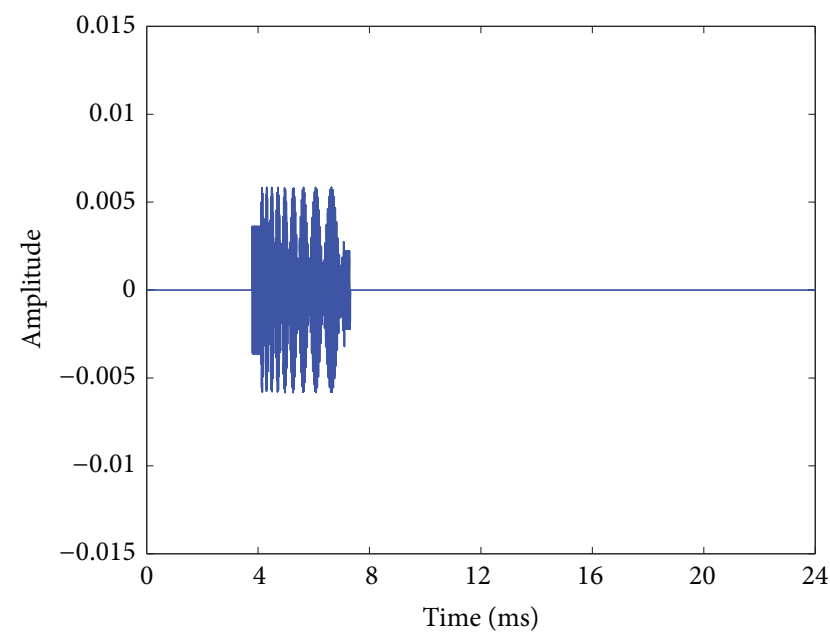

(b)

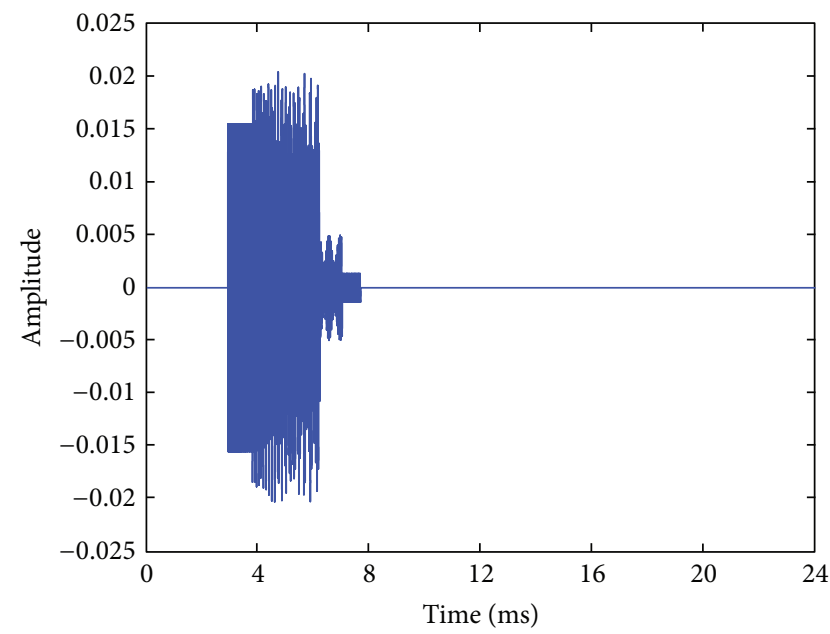

(d)

FIGURE 4: (a) Two overlapping LPM ultrasonic echoes at distances of $0.65 \mathrm{~m}$ and $0.7 \mathrm{~m}$ from a sound source. (b) Estimation of two overlapping LPM ultrasonic echoes. (c) Three overlapping LPM ultrasonic echoes at distances of $0.5 \mathrm{~m}, 0.65 \mathrm{~m}$, and $0.75 \mathrm{~m}$ from a sound source. (d) Estimation of the three overlapping LPM ultrasonic echoes.

the estimated Doppler velocity is illustrated in Figures 1(c) and $1(\mathrm{~d})$. Then, we considered examples of two overlapping LPM ultrasonic echoes, the distances of which, with a sound source, were $0.65 \mathrm{~m}$ and $0.7 \mathrm{~m}$, and three overlapping echoes, the distances of which were $0.5 \mathrm{~m}, 0.65 \mathrm{~m}$, and $0.75 \mathrm{~m}$, as shown in Figure 4, under the additive WGN signal. The Doppler velocities in each object were assumed that the maximum velocity was $5 \mathrm{~m} / \mathrm{s}$ and the minimum velocity was $-5 \mathrm{~m} / \mathrm{s}$. In addition, the maximum distance of the multiple objects is $1 \mathrm{~m}$. The Doppler velocity estimation results of the overlapping LPM ultrasonic echoes are tabulated in Table 1. The SNR under consideration was $10 \mathrm{~dB}$. Table 2 describes the estimation results of the three closed overlapping LPM ultrasonic echoes. In the table, the actual parameters in the simulation are listed. The SNR used to support the Doppler velocity estimation of three overlapping echoes was $22 \mathrm{~dB}$. The step size in both cases for the grid search was $0.05 \mathrm{~m} / \mathrm{s}$.
Ultrasonic velocity measurement by high-resolution Doppler velocity estimation was evaluated with computer simulations using MATLAB. In simulation, the environment was assumed that SNR of the reflected echo was approximately $20 \mathrm{~dB}$ by adding normally distributed random noise. As for the Doppler velocity estimation referred in [4], which requires two cycles of LPM signal, estimation is based on time duration of two peaks in the cross-correlation function. It does not have any problem with only one moving object but when the number of moving objects is more than one object, it is difficult to estimate the Doppler velocities due to many peaks. Hence, the proposed method is designed to estimate the Doppler velocity of two and three objects using only one cycle of LPM signal. However, this is based on EM algorithm and requires convergence to produce at least a local maximum. It is under the iterative procedure in nature. 
TABLE 1: Estimation results for the two overlapping LPM ultrasonic echoes with $10 \mathrm{~dB}$ SNR.

\begin{tabular}{lcc}
\hline LPM ultrasonic signals & First echo & Second echo \\
\hline & -5 & 3.5 \\
Actual velocities $(\mathrm{m} / \mathrm{s})$ & 3.6 & -1.6 \\
& 3.3 & 2 \\
\hline & -4.95 & 3.55 \\
Estimated velocities (m/s) & 3.65 & -1.65 \\
& 3.3 & 1.95 \\
\hline
\end{tabular}

TABLE 2: Estimation results for the three overlapping LPM ultrasonic echoes with $22 \mathrm{~dB}$ SNR.

\begin{tabular}{lccc}
\hline LPM ultrasonic signals & First echo & Second echo & Third echo \\
\hline \multirow{3}{*}{ Actual velocities (m/s) } & 4 & 3.5 & 2 \\
& 1 & -2 & 5 \\
& -4.5 & 3 & -5 \\
\hline \multirow{2}{*}{ Estimated velocities (m/s) } & 4.05 & 3.6 & 2.05 \\
& 1 & -2.1 & 5.1 \\
& -4.45 & 3.1 & -5.15 \\
\hline
\end{tabular}

\section{Conclusion}

In this paper, we first presented the Doppler velocity estimation of the overlapping LPM ultrasonic echoes using the EM algorithm. The single echo results were achieved by the $M$-step of algorithm, that is, the maximum likelihood estimation. The cases of three and two overlapping LPM ultrasonic echoes were addressed by first performing the Doppler velocity estimation of the first echo, followed by the estimation of the Doppler velocities of the residual echoes using the EM algorithm. The grid search was used for the searching engine. Our proposed method was evaluated by performing MATLAB computer simulations. The simulation results confirmed the validity of the proposed method.

\section{Conflict of Interests}

The authors declare that there is no conflict of interests regarding the publication of this paper.

\section{References}

[1] S. Hirata, M. K. Kurosawa, and T. Katagiri, "Cross-Correlation by single-bit signal processing for ultrasonic distance measurement," IEICE Transactions on Fundamentals of Electronics, Communications and Computer Sciences, vol. 91, no. 4, pp. 10311037, 2008.

[2] R. A. Altes, "Sonar velocity resolution with a linear period modulated pulse," The Journal of the Acoustical Society of America, vol. 61, pp. 1019-1030, 1977.

[3] J. J. Kroszczynski, "Pulse compression by means of linear-period modulation," Proceedings of the IEEE, vol. 57, no. 7, pp. 12601266, 1969.

[4] S. Hirata and M. K. Kurosawa, "Ultrasonic distance and velocity measurement using a pair of LPM signals for cross-correlation method: improvement of Doppler-shift compensation and examination of Doppler velocity estimation," Ultrasonics, vol. 52, no. 7, pp. 873-879, 2012.

[5] R. Jacob, T. Thomas, and A. Unnikrishnan, "Application of fractional fourier transform," IEEE Transactions on Ultrasonics, Ferroelectrics and Frequency Control, vol. 57, pp. 2324-2333, 2010.

[6] D. M. J. Cowell and S. Freear, "Separation of overlapping linear frequency modulated (LFM) signals using the fractional fourier transform," IEEE Transactions on Ultrasonics, Ferroelectrics, and Frequency Control, vol. 57, no. 10, pp. 2324-2333, 2010.

[7] S. M. Kay, Fundamentals of Statistical Signal Processing, Prentice Hall, New York, NY, USA, 1993.

[8] R. Demirli and J. Saniie, "Model-based estimation of ultrasonic echoes. Part I: analysis and algorithms," IEEE Transactions on Ultrasonics, Ferroelectrics, and Frequency Control, vol. 48, no. 3, pp. 787-802, 2001. 

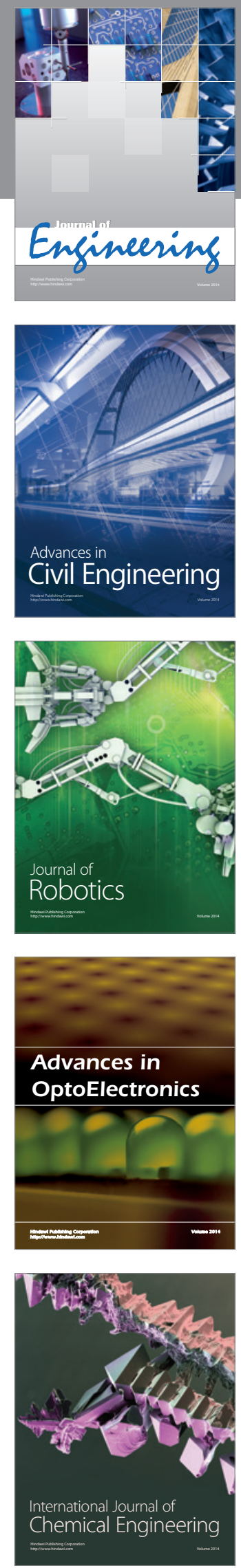

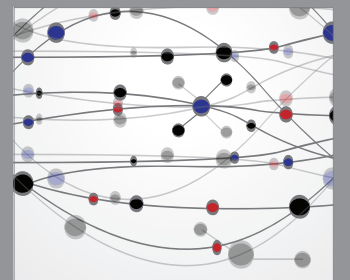

The Scientific World Journal
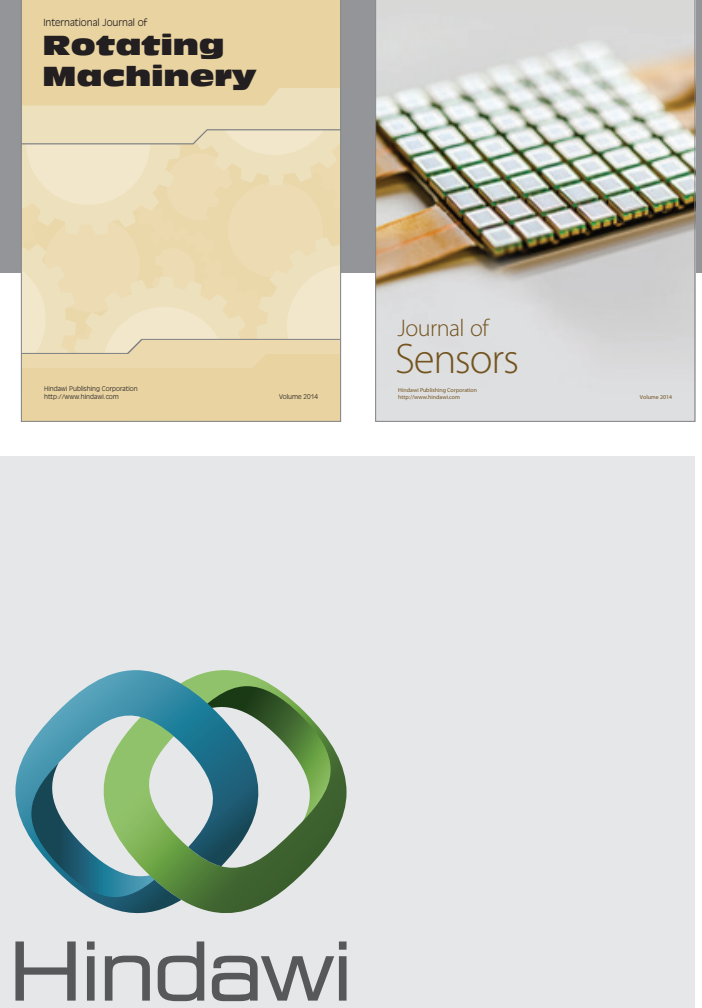

Submit your manuscripts at http://www.hindawi.com
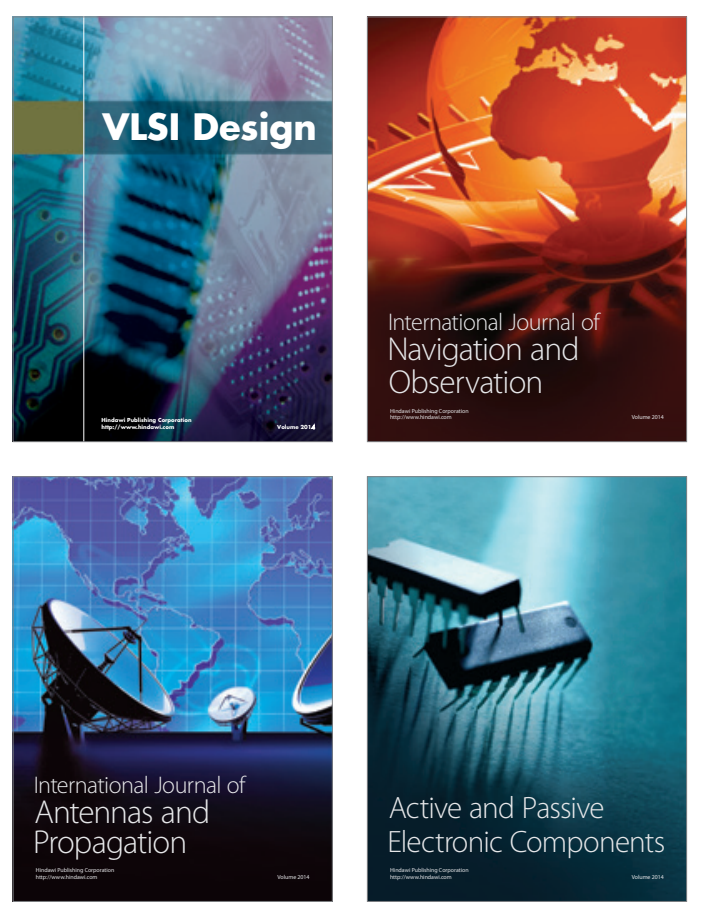
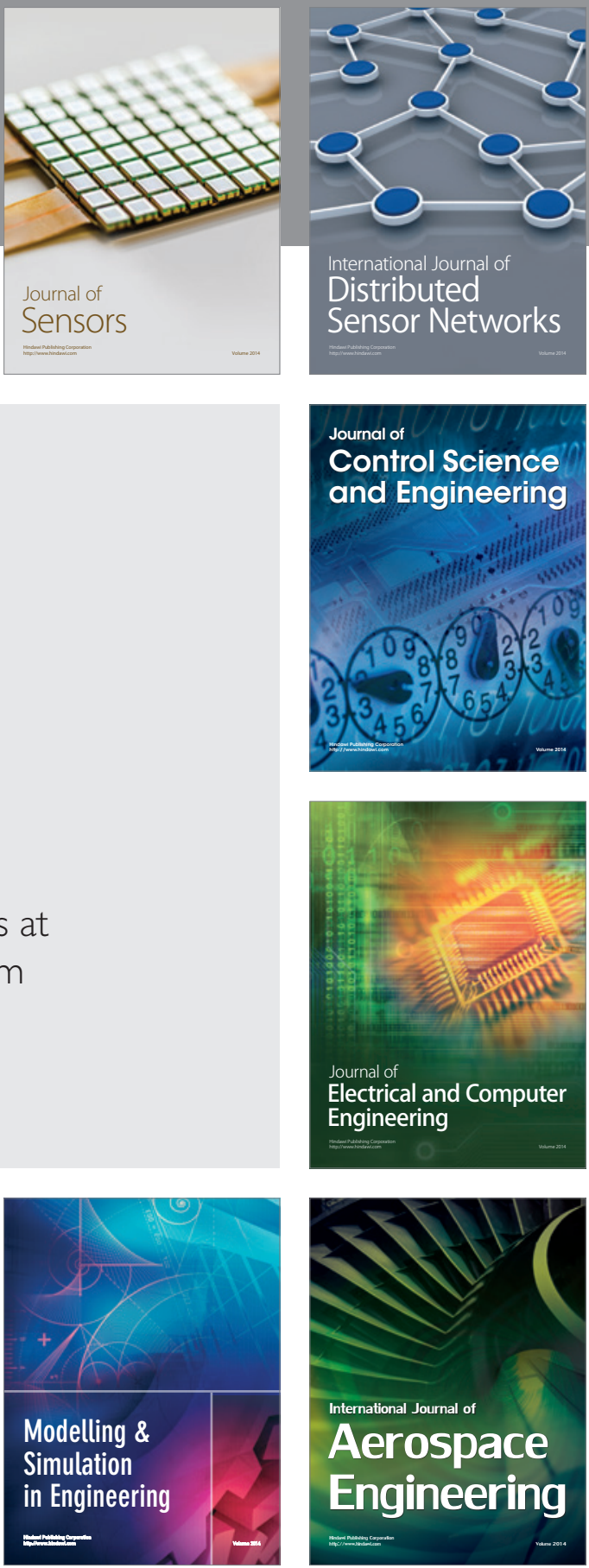

Journal of

Control Science

and Engineering
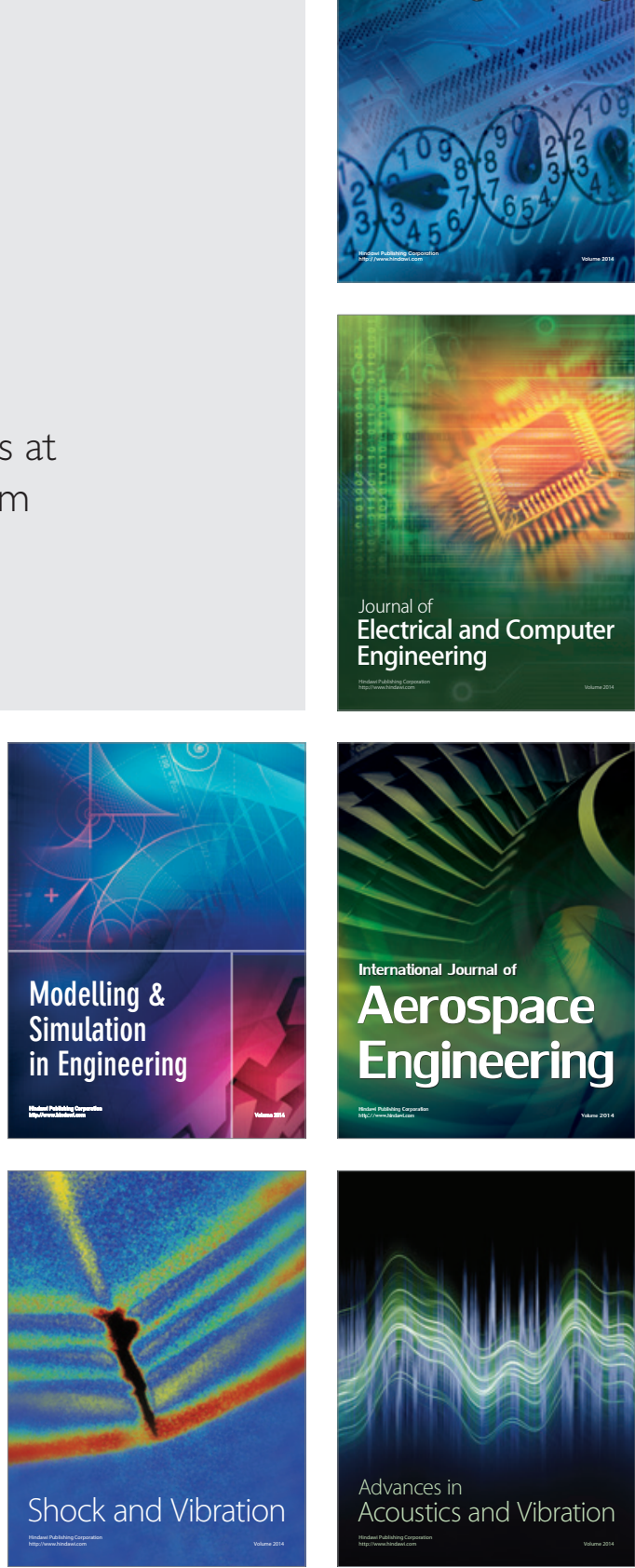